13.4

\title{
Перестраиваемый источник многочастотного излучения Ка-диапазона на основе импульсной гирорезонансной лампы обратной волны
}

\author{
(C) Р.М. Розенталь, С.В. Самсонов, А.А. Богдашов, И.Г. Гачев, А.Н. Леонтьев, Н.С. Гинзбург
}

Институт прикладной физики РАН, Нижний Новгород, Россия

E-mail: rrz@ipfran.ru

Поступило в Редакцию 13 мая 2021 г.

В окончательной редакции 21 июня 2021 г.

Принято к публикации 23 июня 2021 г.

Получены режимы многочастотной генерации в импульсной гирорезонансной лампе обратной волны Ка-диапазона на основе волновода с винтовой гофрировкой. Импульсная мощность излучения составляла $30-35 \mathrm{~kW}$ при длительности импульсов около $120 \mu \mathrm{s}$ и частоте повторения до $10 \mathrm{~Hz}$. За счет изменения величины магнитостатического поля в области взаимодействия и питч-фактора частиц реализована перестройка центральной частоты генерации в диапазоне $32-35.5 \mathrm{GHz}$ и расстояния между основными спектральными линиями в диапазоне $360-550 \mathrm{MHz}$.

Ключевые слова: гиро-ЛОВ, винтовой гофрированный волновод, многочастотные режимы генерации.

DOI: 10.21883/PJTF.2021.19.51509.18868

Одним из применений мощного СВЧ-излучения миллиметрового диапазона является нагрев плазмы в электронно-циклотронных резонансных источниках ионов [1]. Одновременно значительный интерес вызывает применение в таких установках двухчастотного СВЧ-нагрева с целью подавления возникающих в плазме неустойчивостей [2-5]. В работе [6] было экспериментально продемонстрировано, что подобное излучение может быть получено в гирорезонансной лампе обратной волны (гиро-ЛОВ) со спирально-гофрированным волноводом (далее винтовая гиро-ЛОВ) при введении сильных внешних отражений. При этом реализуется так называемый „частотный“ механизм автомодуляции, связанный с одновременным возбуждением нескольких продольных мод совокупной электродинамической системы. Определенным недостатком такого подхода является дискретный характер перестройки расстояния между спектральными компонентами. Как показано далее, непрерывную перестройку соответствующего расстояния в винтовой гиро-ЛОВ можно реализовать и без использования внешних отражений.

Экспериментальные исследования проводились на основе импульсной винтовой гиро-ЛОВ Ка-диапазона. Электронная пушка с реверсом магнитного поля, аналогичная использованной в работе [7], формировала винтовой электронный пучок с энергией $40 \mathrm{keV}$, током $6 \mathrm{~A}$, питч-фактором (отношением поперечной осцилляторной скорости к продольной) до 1.8 , разбросом поперечных скоростей $15-25 \%$ при длительности импульса около $120 \mu \mathrm{s}$ (рис. 1,a). Высоковольтный модулятор обеспечивал частоту повторения импульсов до $10 \mathrm{~Hz}$. Основное магнитное поле величиной $0.5-0.7 \mathrm{~T}$ в области взаимодействия формировалось „теплым“ соленоидом на постоянном токе с жидкостным охлаждением. При этом за счет подстройки тока $I_{k}$ дополнительной катушки со встречным направлением поля, расположенной в области катода (катодной катушки), можно было обеспечивать плавное изменение коэффициента перемагничивания и соответственно питч-фактора электронов от 0.3 до 1.8 (согласно расчетам) при заданном значении тока основного соленоида $I_{s o l}$.

Волновод с трехзаходной винтовой гофрировкой со средним радиусом $3.6 \mathrm{~mm}$, периодом гофрировки $11.6 \mathrm{~mm}$ и длиной $L=240 \mathrm{~mm}$ обеспечивал связь встречной волны $\mathrm{TE}_{1,1}$ и квазикритической моды $\mathrm{TE}_{-2,1}$ и их взаимодействие с электронным пучком на второй гармонике циклотронной частоты. Расчетная групповая скорость рабочей волны в диапазоне частот $30-38 \mathrm{GHz}$ составляла $0.5 c$. Трехдисковое вакуумное окно обеспечивало отражения менее $1 \%$ во всем диапазоне рабочих частот. Выходное излучение преобразовывалось в квазиоптический волновой пучок, порядка $1 \%$ мощности которого отклонялось диэлектрической пластиной в сторону находящегося на некотором расстоянии приемного рупора, соединенного со стандартным (сечением $7.2 \times 3.4 \mathrm{~mm})$ волноводом, так что для СВЧ-диагностики использовался сигнал милливаттного уровня мощности. Основная часть излучения поступала в калориметрическую нагрузку с внутренним калибратором, обеспечивавшую относительную точность измерения около 10\% при уровне средней мощности выше $10 \mathrm{~W}$. Диагностический сигнал поступал на полупроводниковый детектор с временем нарастания $<0.5 \mathrm{~ns}$ и регистрировался цифровым осциллографом с полосой $4 \mathrm{GHz}$ (рис. 1,a). Спектральные измерения проводились методом гетеродинирования путем подачи на осциллограф сигнала на разностной частоте, формируемой смешением сигнала опорного генератора Г4-156 с диаг- 

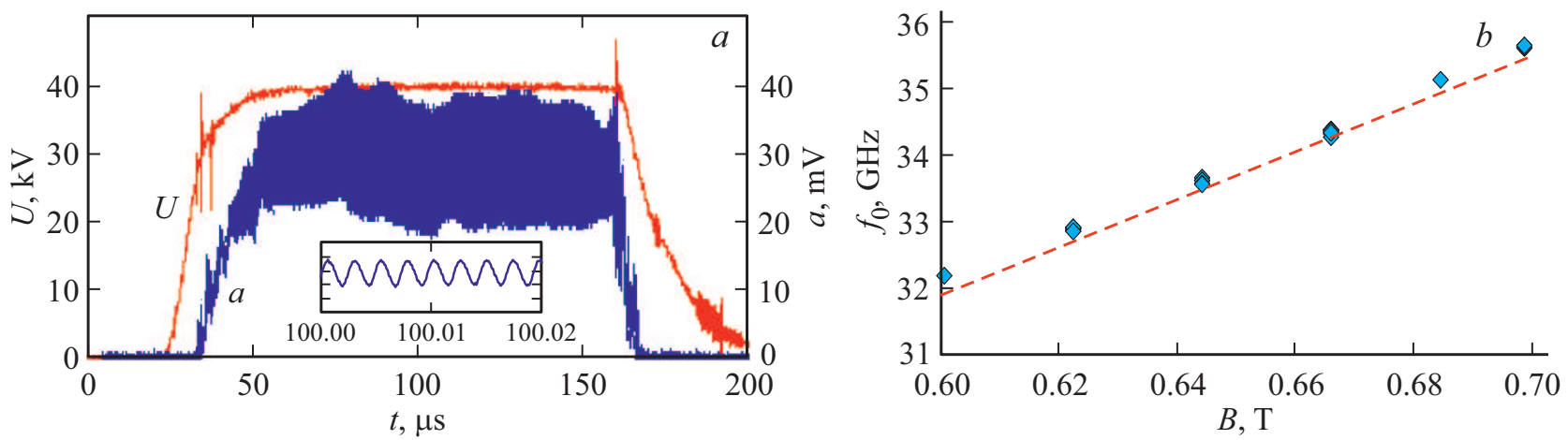

Рис. 1. $a-$ осциллограммы импульса ускоряющего напряжения $U$ и сигнала с детектора $a$ в режиме периодической автомодуляции $(B \approx 0.7 \mathrm{~T}) ; b-$ зависимость центральной частоты генерации от магнитного поля (штриховая линия - расчетная частота точного синхронизма).

ностическим сигналом. Рабочая полоса смесителя составляла $12 \mathrm{GHz}$, частота дискретизации осциллографа составляла $2 \cdot 10^{10}$ выборок в секунду. При изменении магнитного поля осуществлялась подстройка частоты опорного генератора в диапазоне $31-33 \mathrm{GHz}$.

В экспериментах были зарегистрированы устойчивые режимы многочастотной генерации при изменении ведущего магнитного поля от 0.6 до 0.7 Т. Верхнее значение магнитного поля ограничивалось предельным током источника. В свою очередь при приближении к нижней границе мощность генерации значительно уменьшалась, что не позволяло проводить достаточно точные калориметрические измерения. Центральная частота генерации $f_{0}$ с хорошей точностью соответствовала расчетной частоте точного синхронизма (рис. $1, b)$.

На рис. 2 представлены характерные спектры выходного излучения при различных значениях магнитного поля $B$. В большинстве случаев спектр излучения состоял из двух спектральных линий, при этом частота сателлита была выше центральной частоты генерации. Максимальный уровень второй по интенсивности спектральной компоненты достигал $-6 \mathrm{~dB}$. При этом усредненная мощность излучения на вершине импульсов составляла от 20 до $35 \mathrm{~kW}$.

При увеличении питч-фактора электронного пучка, осуществляемого за счет увеличения отношения тока катодной катушки $I_{k}$ к току основного соленоида $I_{s o l}$, наблюдался последовательный запуск стационарных одночастотных колебаний, последующий переход к режиму периодической и далее к режиму хаотической автомодуляции (рис. 3). Было зарегистрировано как минимум одно удвоение периода автомодуляции, последовательность которых является одним из типичных путей перехода к хаосу [8]. Заметим, что аналогичные бифуркации наблюдались ранее и в экспериментах с гиро-ЛОВ на основе гладкого волновода [9].

Одним из наиболее интересных и представляющих практический интерес результатов данных экспериментов, по нашему мнению, является демонстрация возможностей контролируемой перестройки частоты автомоду-
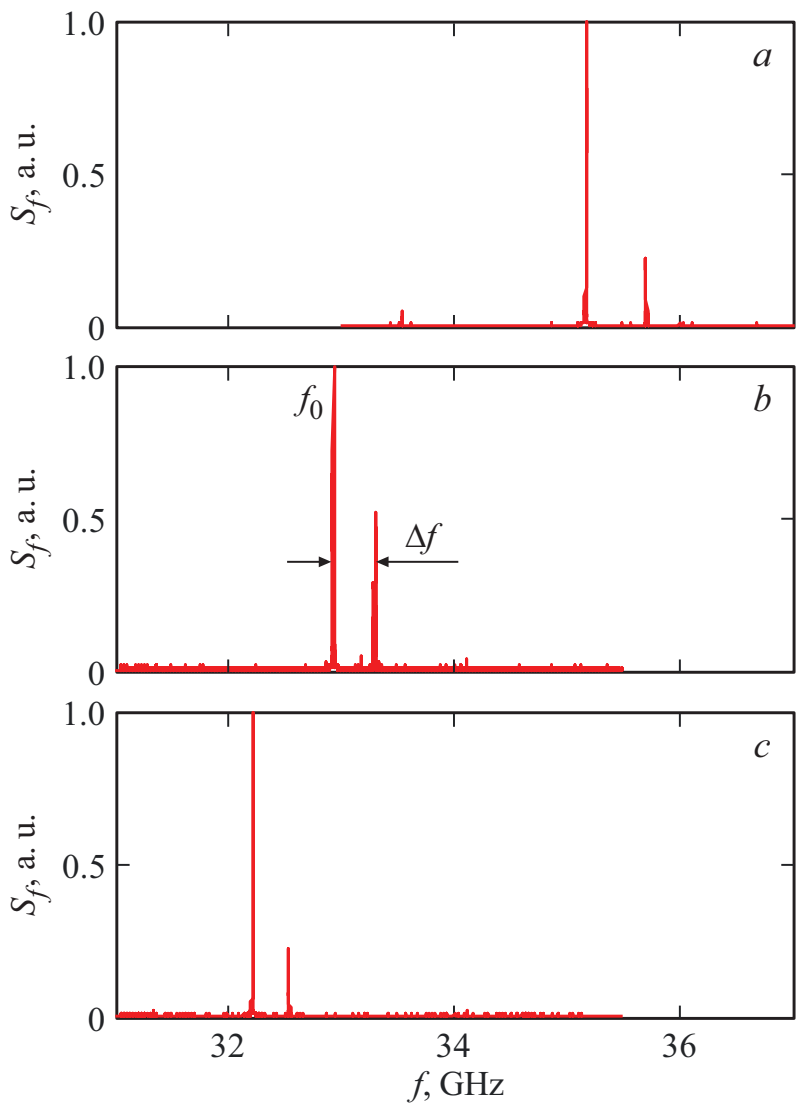

Рис. 2. Спектры выходного излучения при значениях ведущего магнитного поля $B \approx 0.68(a), 0.62(b)$ и $0.6 \mathrm{~T}(c)$.

ляции. Как показали эксперименты, при фиксированном значении тока основного соленоида изменение тока катодной катушки (приводящее, как было отмечено, к изменению питч-фактора частиц) позволяло менять частоту автомодуляции $\Delta f$ в достаточно широких пределах, а именно $360-550 \mathrm{MHz}$ (рис. 3). Отметим, что результаты трехмерного моделирования данной гиро-ЛОВ методом крупных частиц на основе программного пакета 


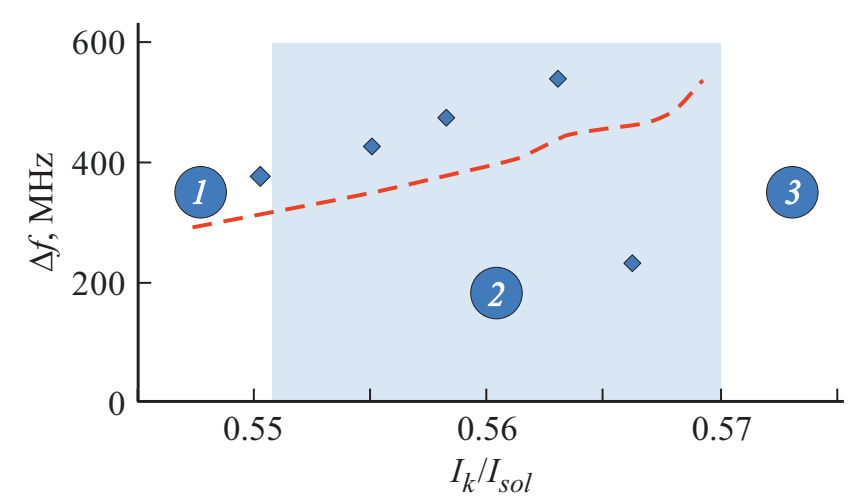

Рис. 3. Зависимость частоты автомодуляции $\Delta f$ от отношения тока катодной катушки к току соленоида при $B \approx 0.68 \mathrm{~T}$ (штриховая линия - расчет, точки - экспериментальные данные). Цифрами обозначены области стационарной генерации (1), периодической автомодуляции (2), удвоения периода и перехода к хаотической автомодуляции (3). Согласно расчетам, при увеличении $I_{k} / I_{\text {sol }}$ от 0.55 до 0.566 питч-фактор частиц меняется от 1 до 1.8 .

CST Studio Suite качественно соответствуют результатам эксперимента: при повышении питч-фактора электронов режим взаимодействия меняется от стационарной одночастотной генерации до автомодуляции со спектром с двумя выраженными частотами, разность между которыми меняется от 300 до $530 \mathrm{MHz}$ (рис. 3, штриховая линия).

В общих чертах динамика исследуемого прибора соответствует свойствам распределенных систем, в которых электронный поток взаимодействует со встречной волной [10], например ЛОВ черенковского типа, однако обнаруженную зависимость частоты автомодуляции от питч-фактора сложно объяснить на основе простейших оценок. Известно, что частота автомодуляции в ЛОВ определяется временем замыкания цепи обратной связи $T=L /\left|v_{g r}\right|+L / v_{\|}$, где $v_{g r}, v_{\|}-$групповая скорость рабочей волны и поступательная скорость электронов. При этом, как показывают расчеты, частота автомодуляции в черенковской ЛОВ примерно в 1.5 раза меньше величины $T^{-1}$ [11-13]. В нашем случае $v_{g r} \approx 0.5 c, v_{\|} \approx(0.2-0.25) c$, что дает значение $T^{-1} \approx 180-220 \mathrm{MHz}$. Таким образом, наблюдаемая частота автомодуляции в винтовой гиро-ЛОВ более чем двукратно превосходит обратное время замыкания цепи обратной связи $\Delta f \geq 2 T^{-1}$. Кроме того, по мере увеличения питч-фактора электронов наблюдается увеличение частоты автомодуляции, хотя, согласно приведенной формуле, увеличение питч-фактора, сопровождающееся уменьшением поступательной скорости электронов, должно приводить к увеличению $T$ и соответственно уменьшению частоты автомодуляции. Таким образом, некоторые особенности генерации в винтовой гиро-ЛОВ существенно отличаются от хорошо изученных эффектов, сопровождающих автомодуляцию в черенковских ЛОВ.
В связи с этим следует обратить внимание на результаты работ [14-16], где возникновение автомодуляции в гиро-ЛОВ на основе гладкого волновода связывается с возбуждением более высоких продольных мод системы. При этом, например, расчетная разность между частотами основной и первой продольной мод составляет величину около $500 \mathrm{MHz}$ для гиро-ЛОВ Ка-диапазона [14], что весьма близко к измеренным в эксперименте величинам $\Delta f$. Это предположение подтверждается также и спектрами излучения, большинство из которых состояло из двух основных спектральных линий (рис. 2).

Таким образом, полученные результаты экспериментальных исследований и „прямого“ трехмерного моделирования не только представляются важными с практической точки зрения, но и стимулируют дальнейшую разработку нестационарной теории и упрощенной модели винтовой гиро-ЛОВ, которая может быть построена на основе уже имеющихся численных моделей винтовой гиротронной лампы бегущей волны [17].

\section{Финансирование работы}

Работа выполнена при поддержке Российского фонда фундаментальных исследований (грант № 19-08-00955), а также частично в рамках госзадания (номер темы 0030-2019-0001).

\section{Конфликт интересов}

Авторы заявляют, что у них нет конфликта интересов.

\section{Список литературы}

[1] В.А. Скалыга, С.В. Голубев, И.В. Изотов, Р.Л. Лапин, С.В. Разин, А.В. Сидоров, Р.А. Шапошников, Прикладная физика, № 1, 17 (2019).

[2] A.G. Shalashov, E.D. Gospodchikov, I.V. Izotov, Plasma Phys. Control. Fusion, 62 (6), 065005 (2020). DOI: $10.1088 / 1361-6587 / \mathrm{ab} 7 \mathrm{f} 98$

[3] V. Toivanen, G. Bellodi, D. Küchler, F. Wenander, O. Tarvainen, Phys. Rev. Accel. Beams, 20 (10), 103402 (2017). DOI: $10.1103 /$ PhysRevAccelBeams.20.103402

[4] R. Rácz, S. Biri, Z. Perduk, J. Pálinkás, D. Mascali, M. Mazzaglia, E. Naselli, G. Torrisi, G. Castro, L. Celona, S. Gammino, A. Galata, JINST, 13 (12), C12012 (2018). DOI: $10.1088 / 1748-0221 / 13 / 12 / \mathrm{C} 12012$

[5] E. Naselli, D. Mascali, M. Mazzaglia, S. Biri, R. Rácz, J. Pálinkás, Z. Perduk, A. Galatá, G. Castro, L. Celona, S. Gammino, G. Torrisi, Plasma Sources Sci. Technol., $28(8)$, 085021 (2019). DOI: 10.1088/1361-6595/ab32f9

[6] Р.М. Розенталь, С.В. Самсонов, А.А. Богдашов, И.Г. Гачев, М.Ю. Глявин, Письма в ЖТФ, 47 (6), 11 (2021). DOI: 10.21883/PJTF.2021.06.50750.18510

[7] S.V. Samsonov, I.G. Gachev, G.G. Denisov, A.A. Bogdashov, S.V. Mishakin, A.S. Fiks, E.A. Soluyanova, E.M. Tai, Y.V. Dominyuk, B.A. Levitan, V.N. Murzin, IEEE Trans. Electron Dev., 61 (12), 4264 (2014). DOI: 10.1109/TED.2014.2364623

[8] M.J. Feigenbaum, Physica D, 7(1-3), 16 (1983). DOI: 10.1016/0167-2789(83)90112-4 
[9] R.M. Rozental, N.S. Ginzburg, M.Y. Glyavin, A.S. Sergeev, IEEE Trans. Microwave Theory Tech., 54 (6), 2741 (2006). DOI: 10.1109/TMTT.2006.874876

[10] Д.И. Трубецков, А.П. Четвериков, Изв. вузов. Прикладная нелинейная динамика, 2 (5), 9 (1994).

[11] Н.С. Гинзбург, С.П. Кузнецов, Т.Н. Федосеева, Изв. вузов. Радиофизика, 21 (7), 1037 (1978).

[12] Н.М. Рыскин, В.Н. Титов, Изв. вузов. Радиофизика, 44 (10), 860 (2001).

[13] С.П. Кузнецов, Изв. вузов. Прикладная нелинейная динамика, 14 (5), 3 (2006).

[14] S.H. Chen, K.R. Chu, T.H. Chang, Phys. Rev. Lett., 85 (12), 2633 (2000). DOI: 10.1103/PhysRevLett.85.2633

[15] S.H. Chen, T.H. Chang, K.F. Pao, C.T. Fan, K.R. Chu, Phys. Rev. Lett., 89 (26), 268303 (2002).

DOI: $10.1103 /$ PhysRevLett.89.268303

[16] A. Grudiev, K. Schünemann, IEEE Trans. Plasma Sci., 30 (3), 851 (2002). DOI: 10.1109/TPS.2002.801610

[17] Н.С. Гинзбург, Р.М. Розенталь, А.С. Сергеев, И.В. Зотова, Письма в ЖТФ, 43 (3), 50 (2017). DOI: $10.21883 /$ PJTF.2017.03.44227.16313 